Chapman University

Chapman University Digital Commons

Engineering Faculty Articles and Research

Fowler School of Engineering

10-17-2019

\title{
vrSensory: Designing Inclusive Virtual Games with Neurodiverse Children
}

Ben Wasserman

Derek Prate

Bryce Purnell

Alex Muse

Kaitlyn Abdo

See next page for additional authors

Follow this and additional works at: https://digitalcommons.chapman.edu/engineering_articles

Part of the Child Psychology Commons, Graphics and Human Computer Interfaces Commons, Other Computer Engineering Commons, and the Other Computer Sciences Commons 


\section{vrSensory: Designing Inclusive Virtual Games with Neurodiverse Children}

\section{Comments}

This is a pre-copy-editing, author-produced PDF of an article accepted for publication in Extended Abstracts of the Annual Symposium on Computer-Human Interaction in Play Companion Extended Abstracts in 2019 following peer review. The definitive publisher-authenticated version is available online at https://doi.org/10.1145/3341215.3356277.

\section{Copyright}

Association for Computing Machinery

Authors

Ben Wasserman, Derek Prate, Bryce Purnell, Alex Muse, Kaitlyn Abdo, Kendra Day, and LouAnne Boyd 


\section{vrSensory: Designing Inclusive Virtual Games with Neurodiverse Children}

\author{
Ben Wasserman \\ Computer Science \\ Chapman University, \\ California, United States \\ wasse114@mail.chapman.ed \\ u \\ Derek Prate \\ Computer Science \\ Chapman University, \\ California, United States \\ prate@chapman.edu

\section{Bryce Purnel} \\ Alex Muse \\ Chapman University, \\ California, United States \\ purne104@mail.chapman.ed \\ amuse@chapman.edu \\ Kaitlyn Abdo \\ Computer Science \\ Chapman University, \\ California, United States \\ kabdo@chapman.edu \\ Kendra Day \\ Computer Science \\ Chapman University, \\ California, United States \\ kday@chapman.edu \\ LouAnne Boyd \\ Computer Science \\ Chapman University, \\ California, United States \\ Iboyd@chapman.edu
}

Permission to make digital or hard copies of part or all of this work for personal or classroom use is granted without fee provided that copies are not made or distributed for profit or commercial advantage and that copies bear this notice and the full citation on the first page. Copyights for third-party components of this work must be honored. For uses, contact the owner/author(s)

CHI 2020 Extended Abstracts, April 25-30, 2020, Honolulu, HI, USA.

(c) 2020 Copyright is held by the owner/author(s).

(C) 2020 Copyright is held by the owner/a

ACM ISBN 978-1-4503-6819-3/20/04.
DOI: https://doi.org/10.1145/3334480.XXXXXXX

*update the above block \& DOI per your rightsreview confirmation (provided after acceptance)

\begin{abstract}
We explore virtual environments and accompanying

interaction styles to enable inclusive play. In designing games for three neurodiverse children, we explore how designing for sensory diversity can be understood through a formal game design framework. Our process reveals that by using sensory processing needs as requirements we can make sensory and social accessible play spaces. We contribute empirical findings for accommodating sensory differences for neurodiverse children in a way that supports inclusive play. Specifically, we detail the sensory driven design choices that not only support the enjoyability of the leisure activities, but that also support the social inclusion of sensory-diverse participants. The participants displayed behaviors in the multi-user version consistent with their behaviors in the single user version with the addition of social behavior. We tie these techniques to game design mechanics to iterate on our efforts to support inclusive game development. Preliminary results are discussed.
\end{abstract}

\section{Author Keywords}

MDA framework; Virtual reality; neurodiversity; sensory processing; inclusion; autism; ADHD; accessibility; assistive technology 


\begin{tabular}{|l|l|l|}
\hline Children & Age & Gender \\
\hline Peter & 8 & Male \\
\hline Cassidy & 11 & Female \\
\hline Max & 11 & Male \\
\hline
\end{tabular}

Table 1. Participant demographics and interests.

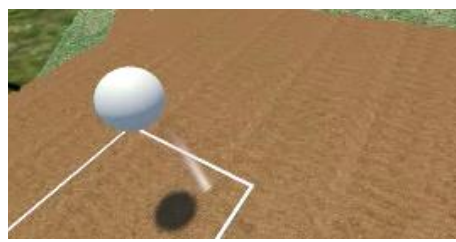

Figure 1: Screenshot of batter's view in baseball game. This game aims to provide a typical level of stimulation and engagement.

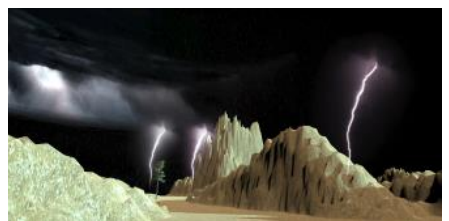

Figure 2: Screenshot of thunderstorm game with lightening and rain features.

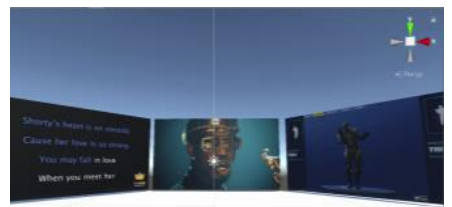

Figure 3: Screenshot of Dance \& Sing Game with three virtual jumbo screens depciting song

\section{CSS Concepts}

- Human-centered computing Human computer interaction (HCI); Accessibility design and evaluation methods; Human-centered computing; Accessibility technologies

\section{Introduction}

Including children with disabilities is a critical issue for the game industry. When designing for children, it is a important to include both players with and without disabilities as the results are better for all players [12]. Children demonstrate varying needs in sensory processing and sensory processing varies widely across players with and without disabilities [6]. Therefore, we engaged a neurodiverse group by enlisting neurotypical children, as well as children with autism or ADHD in the design to ensure the appeals to, and is usable by, children with multiple perspectives [12]. To mediate sensory needs, we employ immersive VR as a platform in order to further level the playing field for neurodiverse players by mediating the sensory experience of leisure activities [3,4,9-11].

Our goal is to discover if we can design $\mathrm{s}$ that result in comfortable environments for a range of children to play independently as well as play together. To explore this aim, we designed three custom virtual reality applications based on extant occupational therapy research regarding four distinct sensory patterns. Specifically, we employed the concept of sensory quadrants described in the Sensory Profile 2 [5]. Then we applied the Mechanics Dynamics and Aesthetics (MDA) framework [7] to describe our specific interaction goals (aesthetics), the runtime play (dynamics) and the underlying features that support these dynamics (the mechanics) as we go through iterative process of design, development, and deployment.

\section{Related Work}

Sensory Processing Challenges

Sensory processing is the organization of sensory information within the body. Sensory input is gathered through all of the senses and integrated in order to inform our actions [2]. For those living with sensory processing challenges these issues are far more than a minor inconvenience and can have substantial impact on daily life. People living with autism are impacted by every sense as well as by the integration of sensory information as required by multi-modal interactions (combining two or more sensory domains such as visual and auditory, temporal, global and local features. Literature regarding ADHD claims that the sensory differences result in several symptoms including somatic complaints, inattention, anxiety, problems with socializing, as well as aggression [8]. Neurotypical children also have diverse sensory patterns that can be leveraged to maximize their comfort and therefore their engagement. Therefore, sensory considerations should be part of mainstream leisure activities--specifically game design. We reflect on games in virtual reality as a potential approach for inclusion, meeting both sensory and social needs of diverse players.

Applying the MDA framework

The MDA framework is a framework that makes the terms and processes of game design and development more formal so that that researchers in these spaces can effectively communicate game criticism, and technical game research. The authors of MDA states that they "believe this methodology will clarify and strengthen the iterative processes of developers, 


\begin{tabular}{|r|rr|}
\multicolumn{1}{l|}{ Child } & Threshold & Interaction \\
\hline Peter & avg & avg \\
Cassidy & high & active \\
Max & high & passive
\end{tabular}

Table 2: Dunn's Sensor Quadrants by threshold and interaction style. These sensory factors impact many aspect

of daily life such as "social

participation, cognition, temperament, and participation" [6]. scholars and researchers alike, making it easier for all parties to decompose, study and design a broad class of game designs and game artifacts"[7]. In this work, we follow their iterative process and employ their vocabulary to understand how addressing sensory needs for children through virtual reality games can be understood more broadly by the player-computer interaction community.

\section{Methods}

We used a convenience sample to recruit three families with children to explore the concepts of personal interests (see Table 1) and sensory profiles that indicate preferences for low to high environmental stimulation and passive to active interaction with the environment. We collected data from Sensory Profile ${ }^{\mathrm{TM}}$ 2 [8] (see Table 2). Across the three child participants, ages $8-11$, (1 female), the children were identified as neurotypical, ADHD, and autistic, respectively. We designed with the children using paper prototypes as well as digital iterations in virtual reality $[1,2]$. In this work, we analyzed the data we collected via interviews with family and children, direct observations during play, video recording and screen capture during single and multiplayer virtual gameplay. We employed the MDA framework to our iterative process.

\section{Developing vrSensory}

Three games were developed in Unity3d with the Steam VR plugin for rendering and Photon Unity Networking for implementing the multi-user aspects. We developed three customized games that tested passive and active interactions related to each participant's sensory profile and interests: baseball for Peter; dance \& sing for Cassidy and thunderstorm for Max.

\section{BASEBALL}

In the baseball game, the batter spawns in at the batter's box (see Figure 1) and is holding a physical baseball bat. When the ball is pitched, the batter can swing to hit the ball and he or she receives textual feedback if the ball was hit, hit foul, or missed. Additionally, the ball slowing down as it approaches the user - and the big hit that results regardless of the force of contact - ensures that the game maintains a passive level of entry for diverse players.

\section{THUNDERSTORM}

The players are spawned into a desert scene, where they can see some hills, a few trees, and rain. There is thunder and rain audio, as well as bolts and flashes of lightning. When a lightning flash occurs, the skybox (sky) lights up with a colorful, cloud-filled stormy image, see Figure 2. The mechanics employed allow each player to teleport, speak to other participants, and adjust the volume of the storm.

\section{DANCE \& SING}

The karaoke contains three massive screens that are lined up in front of and angled towards the participant. On the leftmost screen, the lyrics of the song that is playing are displayed. On the middle screen, a music video of the song is shown; and on the rightmost screen, an avatar is showing dance moves, see Figure 3. Each participant is spawned in to the game next to each other, and they are able to speak to other participants, sing, dance, watch the music video, or do all of the aforementioned.

\section{Analysis}

MDA, a framework focused on the Mechanics, Dynamics, and Aesthetics as a formal approach to 


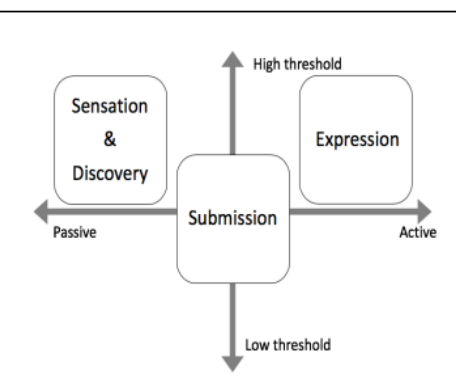

Figure 5: Dunn's quadrants are divided by the axis of self-regulation continuum that spans from passive to active, and the neurological threshold continuum that spans from low to high [5]. In this work we apply these axes to software design by considering passive to active selfregulation in terms of the user's interactions with the system and the low to high neurological thresholds as the intensity of sensory features in the virtual environment.

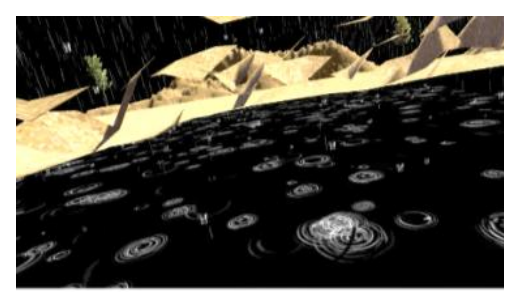

Figure 6: Screenshot of the "black lake" "with water droplets" described when she is inside the mountain. game research [7], allows us to clearly communicate about players and their interactions. We see a mapping between the sensory profiles and the aesthetics of the user experience, see Figure 5 . This suggests a link between sensory patterns and game patterns. Each virtual environment afforded the players a way to express their unique sensory needs - to varying degrees. Next, we detail the mechanics, dynamics and aesthetics we observed. With comfort as the primary goal for user experience, we took particular notice to how they felt as well of the type of experiences they had based on the formal categories from the MDA framework that encourages analysis to be conducted in two directions (i.e., from mechanics creating run-time dynamics that create a certain aesthetic to the other direction where the aesthetics are traced back to mechanics)[7].

\section{Results}

We found that when the participants used single-player games designed for various degrees of passive to active interaction, they displayed behaviors consistent with their sensory profiles. We also found that the aesthetics from the MDA framework map onto the small sample of sensory patterns used to inspire our design. Starting with the aesthetics, we observed aesthetics such Discovery, Fellowship, Submission, and Sensation. We describe the mechanics in Table 3; the specific dynamics in Table 4 and our insights regarding aesthetics that were observed are described below.

\section{Thunderstorm}

Thunderstorm as a single player was experienced as sensation, and discovery [7]. The experiences the children described can be interpreted as sense-pleasure and exploring uncharted territory, which they did in their own ways in spite the of design being intended for the passive profile. The sensation and discovery aesthetics are described below.

SensATION \& Discovery Aesthetics

The Sensation aesthetic provides a sense-pleasure element to the game. The discovery aesthetic provides "uncharted territory" to be discovered. The experiences of sense-pleasure and exploring are evident in the thunderstorm game as the children made various comments directly mentioning their senses and discoveries as they experienced the virtual environment. Cassidy shared her experience using words that describe sensations as she teleported around and explores the scene (see Figure 6):

"What I really liked about thunderstorm was you could go inside this rock and you could see the water droplets come inside the rock and drops on the floor... It's like a black lake"

As might be expected of her active profile, she explored the entire game world relatively quickly. She also used her physical body movements to explore the virtual world, as expected of an active profile. She explored the terrain as she spins her body around to observe the lightning storm, and she teleported inside trees and mountains. Peter has a typical sensory profile and said: "I liked watching the rain and hearing the thunder". Peter mentioned the auditory and visual sensations of the game, but he displayed a neutral level of interest (e.g., not excited and not bored) for this game designed for the passive sensory profile. He moved around virtually in the thunderstorm game. He maked the game more active through unintended in-game actions by shooting light beams from the controller. He 


\section{Mini Game Mechanic that are \\ Baseball \\ thrown \\ automatically \\ Thunder(audio) and Lightning \\ in areas if \\ dessert \\ mountain \\ scape, \\ adjustable \\ Thunderstorm \\ volume, chat \\ functionality \\ -Multiple \\ jumbotrons with different simultaneous representations \\ of songs \\ (lyrics, \\ dancing, singer video)}

Table 3: Planned Mechanics from first multi-player study. also teleported around, while he relaxed in the physical world by sitting and kneeling on the floor. Max explored the discovery aesthetic through verbally describing the physical environment:

"Icicles, icicles, rain - I saw the rain! That's cool. That's cool, that I saw the rain, whole bunch thunder right now. Loud, I like that"

Baseball

SUBMISSION AESTHETIC

The submission aesthetic is surrendering to play for play's sake. The sporting nature of the baseball game resulted in predictable behavior by the players. Players bat against the machine pitcher. The gameplay in this popular American pastime [7] was immediately evident. The children commented about the degree of challenge as they interpreted the goal of batting as to beat the pitcher by making a big hit. This challenge dynamic was planned through the mechanics that make the ball slow down as it approaches the player. Here we see the impact of the challenge dynamic on Max as he exclaimed that he hit the ball "way up to the moon." Max was delighted and maintained a high level of engagement. Max's sister reported that the 15 minutes we permitted him to play as a batter was one of the longest times he has stayed in engaged in a sporting activity. The dynamic of slowing down the ball when a player is in the batter's box allowed entry for the passive sensory profile. Alternatively, the average and high-threshold players reported to be slightly turned off by the ease of use, yet neither asked them to come out sooner than the designated 15 minutes. After play, Peter said: "Hard, fun, didn't have to do much for it (the ball) to go far, liked how ball slowed, but make hit more realistic based on swing". Cassidy stated that the baseball game was: "Fun, glitchy, (the ball) would go far no matter what. I didn't like that the ball slowed down but liked that is was most active". These descriptions of the submission aesthetic confirm we have created a comfortable play space for each sensory profile and now can iterate on the mechanics to increase the challenge for those who want a more of an active game.

\section{Dance \& Sing}

EXPRESSION AESTHETIC

The expression aesthetic is a "game about selfdiscovery" [7]. Cassidy was more reserved in her dancing and singing in the lab study than in the video of her home paper prototyping session in [2]. Max tried the dance \& sing VR prototype in his home and did dance in spite of the presence of the research team. Cassidy also reported that the dance $\&$ sing game is a bit boring as there was only one dance move. She liked that the dance moves were shown, that there was a music video, and that the lyrics were displayed, but she reported there needs to be more dances from which to choose. Max also requested more songs be added to the short selection that had been prepared for the study. Peter did not choose to try the dance \& sing game. Again, the expression aesthetic revealed the need for more ways to express, so we will add mechanics to support more expression in the future.

In summary, our goal for the single player VR prototypes was to understand the aesthetics and to confirm if the environments were comfortable and enjoyable for the primary player. After viewing the players, we iterated on the to add a social component. Next, we provide the results with multiple players networked into the same game. 


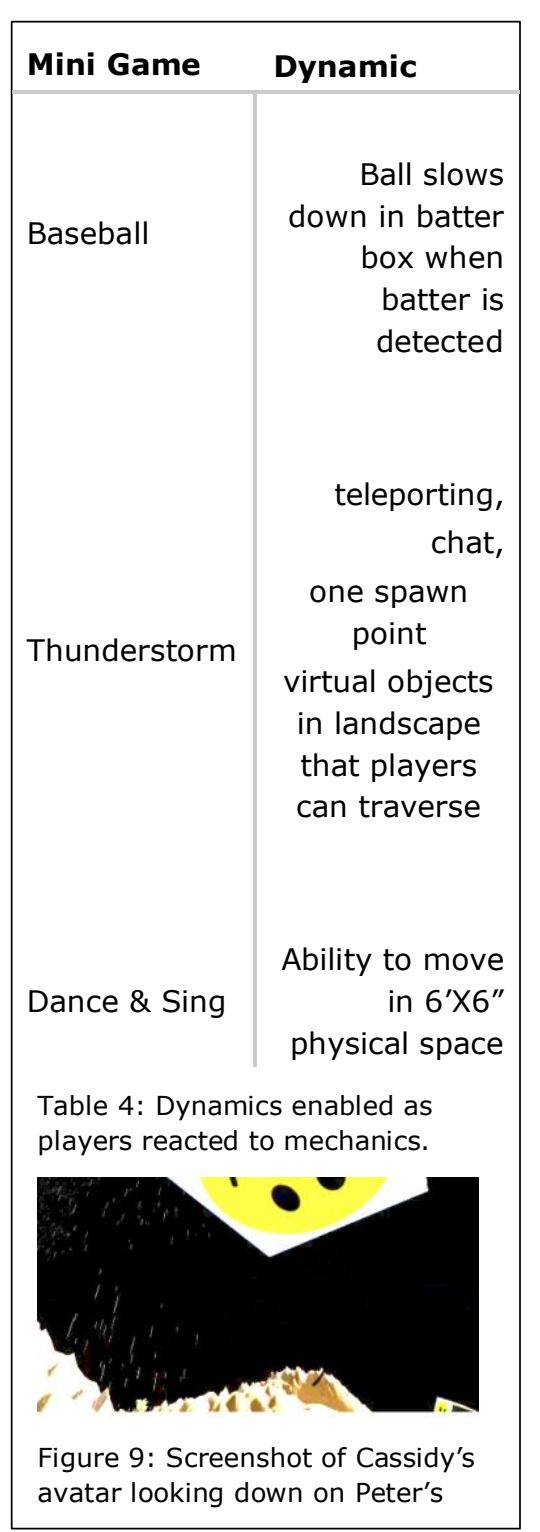

The final user study for this work had all the children come together to use the games in multi user mode. We achieved varying degrees of networked capabilities across the games. We prioritized the most passive sensory pattern by first building a networked mode where all three children could be in Thunderstorm together and had the ability to chat using their voices. When spawned into the multi user game, the three children could see the others as a smiley face box (see Figure 8), use voice chat to communicate with each other and use the controllers to move and teleport around the area. They could also move about in the six-foot by six-foot physical space.

\section{Fellowship Aesthetic}

The Fellowship aesthetic is the experience elicited from a "game as social framework" [7]. When all three users were in Thunderstorm together, they explored

together. Max instantly connects with the others. Max said, "You hear that too, Peter? It's really loud". They started communicating about their respective positions and rapidly changed them via teleportation. All three players started to move in an organized fashion. For example, Cassidy moved away and said, "Meet me at the tree." The boys also teleported away and chased after each other. When all three children did this, they pointed the laser at the others' avatar (in order to teleport), thus landing on top of him or her. Cassidy said, "You found me. Why are you guys on top of each other? Now I am on top". Her avatar spawned on top of his, see Figure 9.

These preliminary results indicate that games designed predominantly for a child with a passive interaction style and high threshold to stimuli can be enjoyable for them as well as appropriated in unique ways to meet the needs of children with different sensory profiles. Thus, each adapted or were accommodated by supporting their sensory needs and when in a networked, they each were social (e.g., talked about their whereabouts as well as the whereabouts of others).

\section{Discussion}

The MDA framework and its mapping to sensory patterns can help developers build experience driven games (as the MDA authors mention). Linking the formal MDA approach to our sensory patterns allows developers and designers to take into account not only how their mechanics and dynamics may elicit certain aesthetics, but how each aesthetic may map to certain sensory preferences. This gives developers the ability to try to design more sensory inclusive games that will still elicit the same aesthetics. For example,

Thunderstorm is a game designed for a passive sensory profile. In our qualitative analysis, we can see that the thunderstorm game elicited aesthetics of sensation and discovery. We may need to take extra consideration into how we will make the game inclusive towards active, experience driven players. We can do that considering certain aesthetics and since we have an idea of how those aesthetics map to sensory patterns, we can design sensory inclusive games that are "fun" or elicit desired aesthetics for all players.

\section{Acknowledgements}

We thank the many students who helped build this game and the families that participated over the course of an academic year. We thank MLAT for the lab space. This work was conducted under IRB protocol \#18-79. 


\section{References}

1. LouAnne Boyd. 2019. Designing SensoryInclusive Virtual Play Spaces for Children. In Proceedings of the 18th ACM International Conference on Interaction Design and Children (IDC '19), 446-451.

2. LouAnne Boyd, Kendra Day, Ben Wasserman, Kaitlyn Abdo, Gillian Hayes, and Erik Linstead. 2019. Paper Prototyping Comfortable VR Play for Diverse Sensory Needs. In Extended Abstracts of the $2019 \mathrm{CHI}$ Conference on Human Factors in Computing Systems (CHI EA '19), LBW1714:1-LBW1714:6.

https://doi.org/10.1145/3290607.3313080

3. LouAnne E. Boyd. 2018. Designing and Evaluation Alternative Channels: Visualizing Nonverbal Communication in AR and VR for People with Autism. University of California, Irvine.

4. LouAnne E. Boyd, Kendra Day, Natalia Stewart, Kaitlyn Abdo, Kathleen Lamkin, and Erik Linstead. 2018. Leveling the Playing Field: Supporting Neurodiversity via Vitual Realities. Technology and Innovation 20.

5. Winnie Dunn. 2014. Sensory ProfileTM 2. Pearson, Bloomington, MN.

6. Winnie Dunn, Lauren Little, Evan Dean, Sara Robertson, and Benjamin Evans. 2016. The State of the Science on Sensory Factors and Their Impact on Daily Life for Children: A Scoping Review.

7. Robin Hunicke, Marc LeBlanc, and Robert Zubek. 2004. MDA: A formal approach to game design and game research. In Proceedings of the AAAI Workshop on Challenges in Game AI, 1722.

8. Lauren M. Little, Evan Dean, Scott Tomchek, and Winnie Dunn. 2017. Sensory Processing Patterns in Autism, Attention Deficit

Hyperactivity Disorder, and Typical Development. Physical \& Occupational Therapy In Pediatrics 0, 0: 1-12.

9. Deysi Helen Ortega, Franceli Linney Cibrian, and Mónica Tentori. 2015. BendableSound: A Fabric-based Interactive Surface to Promote Free Play in Children with Autism. In Proceedings of the 17th International ACM SIGACCESS Conference on Computers \& Accessibility (ASSETS '15), 315-316.

10. Kathryn E. Ringland, Christine T. Wolf, LouAnne E. Boyd, Mark S. Baldwin, and Gillian R. Hayes. 2016. Would You Be Mine: Appropriating Minecraft As an Assistive Technology for Youth with Autism. In Proceedings of the 18th International ACM SIGACCESS Conference on Computers and Accessibility (ASSETS '16), 33-41.

11. Kathryn E. Ringland, Rodrigo Zalapa, Megan Neal, Lizbeth Escobedo, Monica Tentori, and Gillian R. Hayes. 2014. SensoryPaint: a multimodal sensory intervention for children with neurodevelopmental disorders. 873-884.

12. Kristen Shinohara, Cynthia L. Bennett, Wanda Pratt, and Jacob O. Wobbrock. 2018. Tenets for Social Accessibility: Towards Humanizing Disabled People in Design. ACM Transactions on Accessible Computing 11, 1: 1-31. 